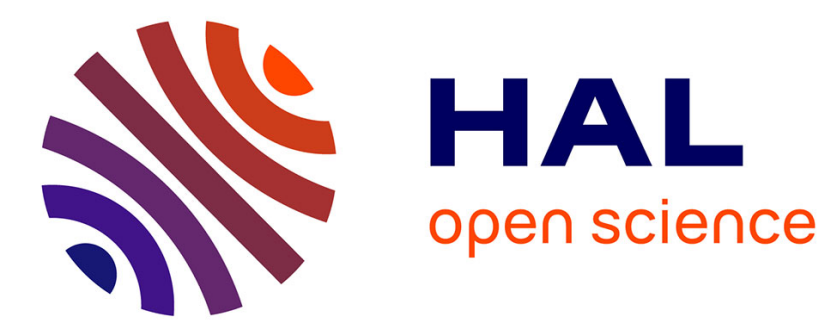

\title{
An intermediate Baum-Katz theorem
}

\author{
Allan Gut, Ulrich Stadtmüller
}

\section{To cite this version:}

Allan Gut, Ulrich Stadtmüller. An intermediate Baum-Katz theorem. Statistics and Probability Letters, 2011, 81 (10), pp.1486. 10.1016/j.spl.2011.05.008 . hal-00770344

\section{HAL Id: hal-00770344 https://hal.science/hal-00770344}

Submitted on 5 Jan 2013

HAL is a multi-disciplinary open access archive for the deposit and dissemination of scientific research documents, whether they are published or not. The documents may come from teaching and research institutions in France or abroad, or from public or private research centers.
L'archive ouverte pluridisciplinaire HAL, est destinée au dépôt et à la diffusion de documents scientifiques de niveau recherche, publiés ou non, émanant des établissements d'enseignement et de recherche français ou étrangers, des laboratoires publics ou privés. 


\section{Accepted Manuscript}

An intermediate Baum-Katz theorem

Allan Gut, Ulrich Stadtmüller

PII:

S0167-7152(11)00183-0

DOI:

10.1016/j.spl.2011.05.008

Reference: $\quad$ STAPRO 6005

To appear in: Statistics and Probability Letters

Received date: 7 March 2011

Revised date: 18 May 2011

Accepted date: 18 May 2011

Please cite this article as: Gut, A., Stadtmüller, U., An intermediate Baum-Katz theorem.

Statistics and Probability Letters (2011), doi:10.1016/j.spl.2011.05.008

This is a PDF file of an unedited manuscript that has been accepted for publication. As a service to our customers we are providing this early version of the manuscript. The manuscript will undergo copyediting, typesetting, and review of the resulting proof before it is published in its final form. Please note that during the production process errors may be discovered which could affect the content, and all legal disclaimers that apply to the journal pertain. 


\title{
An intermediate Baum-Katz theorem
}

\author{
Allan Gut \\ Uppsala University
}

\author{
Ulrich Stadtmüller \\ University of Ulm
}

\begin{abstract}
We extend the classical Hsu-Robbins-Erdős theorem to the case when all moments exist, but the moment generating function does not, viz., we assume that $E \exp \left\{\left(\log ^{+}|X|\right)^{\alpha}\right\}<\infty$ for some $\alpha>1$. We also present multiindex versions of the same and of a related result due to Lanzinger in which the assumption is that $E \exp \left\{|X|^{\alpha}\right\}<\infty$ for some $\alpha \in(0,1)$.
\end{abstract}

\section{Introduction}

In the seminal paper Hsu and Robbins (1947) the authors introduced the concept of complete convergence, and proved that the sequence of arithmetic means of independent, identically distributed (i.i.d.) random variables converges completely (which means that the Borel-Cantelli sum of certain tail probabilities converges) to the expected value of the summands, provided their variance is finite. The necessity was proved by Erdös $(1949,1950)$. This result was later extended in a series of papers which culminated in the now classical paper Baum and Katz (1965), in which the equivalence of (1.1), (1.2), and (1.4) in the following theorem was demonstrated.

Theorem 1.1 Let $r>0, \alpha>1 / 2$, and $\alpha r \geq 1$. Suppose that $X, X_{1}, X_{2}, \ldots$ are i.i.d. random variables with partial sums $S_{n}=\sum_{k=1}^{n} X_{k}, n \geq 1$. If

$$
E|X|^{r}<\infty \quad \text { and, if } r \geq 1, \quad E(X)=0,
$$

then

$$
\begin{aligned}
& \sum_{n=1}^{\infty} n^{\alpha r-2} P\left(\left|S_{n}\right|>n^{\alpha} \varepsilon\right)<\infty \quad \text { for all } \varepsilon>0 \\
& \sum_{n=1}^{\infty} n^{\alpha r-2} P\left(\max _{1 \leq k \leq n}\left|S_{k}\right|>n^{\alpha} \varepsilon\right)<\infty \quad \text { for all } \varepsilon>0 .
\end{aligned}
$$

If $\alpha r>1$ we also have

$$
\sum_{n=1}^{\infty} n^{\alpha r-2} P\left(\sup _{k \geq n}\left|S_{k} / k^{\alpha}\right|>\varepsilon\right)<\infty \quad \text { for all } \varepsilon>0 .
$$

Conversely, if one of the sums is finite for all $\varepsilon>0$, then so are the others (for appropriate values of $r$ and $\alpha), E|X|^{r}<\infty$ and, if $r \geq 1, E(X)=0$.

REMARK 1.1 Strictly speaking, if one of the sums is finite for some $\varepsilon>0$, then so are the others (for appropriate values of $r$ and $\alpha$ ), and $E|X|^{r}<\infty$. However, we need convergence for all $\varepsilon>0$ in order to infer that $E(X)=0$ for the case $r \geq 1$. The same remark applies to Theorem 3.1 below.

AMS 2000 subject classifications. Primary 60F15, 60G50, 60G60; Secondary 60F05.

Keywords and phrases. Sums of i.i.d. random variables, convergence rates, law of large numbers, almost exponential moments, random fields, multiindex.

Abbreviated title. An intermediate Baum-Katz theorem.

Date. May 18, 2011 
The Hsu-Robbins-Erdös part of the theorem thus concerns (1.1) $p=1$. Spitzer (1956) and Katz (1963) proved the same equivalence for the cases $r=p=1$ and $r>0, p=1$, respectively (with a small exception in the latter case). The equivalence (in the above sense) between (1.1), (1.2) and (1.4) is precisely the main theorem in Baum and Katz (1965) as mentioned prior to the statement of the result. Finally, the equivalence between (1.1) and (1.3) was proved differently in Chow (1973); note, however, that $(1.3) \Longrightarrow(1.2)$ is trivial and, given the Baum-Katz theorem, the converse follows (essentially) via the Lévy inequalities.

For $p=2$ one falls into the realm of the central limit theorem, so there is no hope for a positive result, since the probabilities do not tend to zero. To compensate for this one may consider probabilites such as $P\left(\left|S_{n}\right|>\varepsilon \sqrt{n \log n}\right)$ and $P\left(\left|S_{n}\right|>\varepsilon \sqrt{n \log \log n}\right)$ in the above sums. The first paper in this direction was Lai (1974). Multiindex versions of much of this can be found in Gut $(1978,1980)$.

Theorem 1.1 provides conditions for the rate of decrease to zero as $n \rightarrow \infty$ of the various tail probabilities. Otherwise put, the theorem provides information about the rate of convergence in the law of large numbers. An alternative question, that will not addressed in this paper, is to ask for the rate at which the tail probabilities tend to 1 as $\varepsilon \searrow 0$. Toward that end, Heyde (1975) proved that

$$
\lim _{\varepsilon \searrow 0} \varepsilon^{2} \sum_{n=1}^{\infty} P\left(\left|S_{n}\right| \geq \varepsilon n\right)=E X^{2},
$$

whenever $E X=0$ and $E X^{2}<\infty$. Remaining values of $r$ and $p$ have later been taken care of in Chen (1978), Spătaru (1999) and Gut and Spătaru (2000). There also exist analogs related, for example, to randomly indexed partial sums, renewal theory, and records; cf. e.g. Gut (2007), Chapters 6 and 7 , and further references given there.

A fair amount of papers have, since then, been devoted to replacing the powers of $n$ in these results by more general weights, and also by introducing dependence assumptions. The proofs of those results generally follow (approximately) the same lines as the proofs of those cited above.

A natural next question is: What can be said about rates growing faster than polynomially?

Theorem 1.2 Let $0<\alpha<1$, and suppose that $X, X_{1}, X_{2}, \ldots$ are i.i.d. random variables with $E(X)=0$ and partial sums $S_{n}=\sum_{k=1}^{n} X_{k}, n \geq 1$. If

$$
E \exp \left\{|X|^{\alpha}\right\}<\infty,
$$

then

$$
\begin{aligned}
& \sum_{n=1}^{\infty} \exp \left\{n^{\alpha}\right\} \cdot n^{\alpha-2} P\left(\left|S_{n}\right|>n \varepsilon\right)<\infty \quad \text { for all } \varepsilon>1 \\
& \sum_{n=1}^{\infty} \exp \left\{n^{\alpha}\right\} \cdot n^{\alpha-2} P\left(\max _{1 \leq k \leq n}\left|S_{k}\right|>n \varepsilon\right)<\infty \quad \text { for all } \varepsilon>1 \\
& \sum_{n=1}^{\infty} \exp \left\{n^{\alpha}\right\} \cdot n^{\alpha-2} P\left(\sup _{k \geq n}\left|S_{k} / k\right|>\varepsilon\right)<\infty \quad \text { for all } \varepsilon>1 .
\end{aligned}
$$

Conversely, if one of the sums is finite for some $\varepsilon>0$, then so are the others, and $E \exp \left\{\left|X / \varepsilon^{\prime}\right|^{\alpha}\right\}<\infty$ for any $\varepsilon^{\prime}>\varepsilon$.

The equivalence of (1.5) and (1.6) is due to Lanzinger (1998) (in a slightly stronger form in that he treats the two tails separately with a somewhat more general moment condition). The implications $(1.7) \Longrightarrow(1.6)$ and $(1.8) \Longrightarrow(1.6)$ are trivial, $(1.6) \Longrightarrow(1.7)$ follows, again, from the Lévy inequalities (see e.g. Gut (2007), Section 3.7), and (1.6) $\Longrightarrow$ (1.8) follows via a refinement of the "slicing device" of Baum and Katz (1965) as given in Lai (1974), page 439, the details of which we omit.

The aim of the present paper is to close the gap between the two results, that is, we consider the case when exponential moments of some power of $\log |X|$ of order larger than one is finite. This will be achieved in the following section. In Section 3 we present multiindex versions of our theorem and of Lanzinger's result. We close with some remarks. 


\section{Main theorem}

Before we present our main result, here are some minor pieces of notation. For $x>0$ we set $\log ^{+} x=\max \{1, \log x\}$. For simplicty and convenience we shall abuse this notation in the sense that we tacitly interpret logarithms as if there were the extra +-sign in running text and in computations. Moreover, $c$ will denote numerical constants whose value are without importance, and, in addition, may change between appearances.

Theorem 2.1 Let $\alpha>1$, and suppose that $X, X_{1}, X_{2}, \ldots$ are i.i.d. random variables with partial sums $S_{n}=\sum_{k=1}^{n} X_{k}, n \geq 1$. If

$$
E \exp \left\{\left(\log ^{+}|X|\right)^{\alpha}\right\}<\infty \quad \text { and } \quad E(X)=0 \text {, }
$$

then

$$
\begin{aligned}
& \sum_{n=1}^{\infty} \exp \left\{(\log n)^{\alpha}\right\} \frac{(\log n)^{\alpha-1}}{n^{2}} P\left(\left|S_{n}\right|>n \varepsilon\right)<\infty \quad \text { for all } \varepsilon>1 ; \\
& \sum_{n=1}^{\infty} \exp \left\{(\log n)^{\alpha}\right\} \frac{(\log n)^{\alpha-1}}{n^{2}} P\left(\max _{1 \leq k \leq n}\left|S_{k}\right|>n \varepsilon\right)<\infty \quad \text { for all } \varepsilon>1 ; \\
& \sum_{n=1}^{\infty} \exp \left\{(\log n)^{\alpha}\right\} \frac{(\log n)^{\alpha-1}}{n^{2}} P\left(\sup _{k \geq n}\left|S_{k} / k\right|>\varepsilon\right)<\infty \quad \text { for all } \varepsilon>1 .
\end{aligned}
$$

Conversely, if one of the sums is finite for some $\varepsilon>0$, then so are the others, and $E \exp \left\{(1-\delta)\left(\log ^{+}|X|\right)^{\alpha}\right\}<\infty$ for any $\delta>0$.

REMARK 2.1 The reason that one obtains a lower bound for $\varepsilon$ in Theorem 1.2 is due to the fact that $\varepsilon$ acts as a scaling parameter there, whereas it is in between a scaling factor and being irrelevant in Theorem 2.1.

REMARK 2.2 If (2.2) holds with $\varepsilon<1 / 2$, then we have, in fact, that $E \exp \left\{\left(\log ^{+}|X|\right)^{\alpha}\right\}<\infty$.

Proof. The general pattern of the proof differs slightly from the usual ones in the area, in that, whereas one typically requires one truncation in LLN-related results and two truncations plus exponential inequalities in LIL-related results, our proof is of the latter kind in spite of the fact that we are in the LLN-domain.

The hard(est) part is

$\mathbf{( 2 . 1 )} \Longrightarrow \mathbf{( 2 . 2 ) :}$ Let $0<\delta<1$ and $\varepsilon>0$ be arbitrary, set, for $n \geq 1$,

$$
b_{n}=\frac{\epsilon n}{(\log n)^{\alpha}} \quad \text { and } \quad c_{n}=n \varepsilon(1-\delta),
$$

define, for $1 \leq k \leq n$,

$$
X_{k}^{\prime}=X_{k} I\left\{\left|X_{k}\right| \leq b_{n}\right\}, \quad X_{k}^{\prime \prime}=X_{k} I\left\{b_{n}<\left|X_{k}\right|<c_{n}\right\}, \quad X_{k}^{\prime \prime \prime}=X_{k} I\left\{\left|X_{k}\right| \geq c_{n}\right\},
$$

and let all objects with primes or multiple primes refer to the respective truncated summands (and recall from above that $\log n=\log ^{+} n$ throughout our computations).

Next, set $A_{n}=\left\{\left|S_{n}\right|>n \varepsilon\right\}$,

$$
\begin{aligned}
& A_{n}^{\prime}=\left\{\left|S_{n}\right|>n \varepsilon \text { and } X_{k}^{\prime \prime} \neq 0 \text { for at most one } k \leq n \text { and } X_{k}^{\prime \prime \prime}=0 \text { for all } k \leq n\right\}, \\
& A_{n}^{\prime \prime}=\left\{X_{k}^{\prime \prime} \neq 0 \text { for at least two } k \leq n\right\}, \\
& A_{n}^{\prime \prime \prime}=\left\{X_{k}^{\prime \prime \prime} \neq 0 \text { for at least one } k \leq n\right\} .
\end{aligned}
$$

We furthermore split $A_{n}^{\prime}$ into $A_{n, 1}^{\prime} \cup A_{n, 2}^{\prime}$, where

$$
\begin{aligned}
& A_{n, 1}^{\prime}=\left\{\left|S_{n}\right|>n \varepsilon \text { and }\left|X_{k}\right| \leq b_{n} \text { for all } k \leq n\right\}, \\
& A_{n, 2}^{\prime}=\left\{\left|S_{n}\right|>n \varepsilon \text { and }\left|X_{k}\right|>b_{n} \text { for exactly one } k \leq n\right\},
\end{aligned}
$$


and note that

$$
A_{n} \subset A_{n, 1}^{\prime} \cup A_{n, 2}^{\prime} \cup A_{n}^{\prime \prime} \cup A_{n}^{\prime \prime \prime}
$$

which tells us that

$$
P\left(\left|S_{n}\right|>n \varepsilon\right)=P\left(A_{n}\right) \leq P\left(A_{n, 1}^{\prime}\right)+P\left(A_{n, 2}^{\prime}\right)+P\left(A_{n}^{\prime \prime}\right)+P\left(A_{n}^{\prime \prime \prime}\right) .
$$

- $P\left(A_{n, 1}^{\prime}\right)$

Since truncation destroys centering, it follows, using standard procedures, that

$$
\begin{aligned}
\left|E S_{n}^{\prime}\right| & =\left|n E X_{k} I\left\{\left|X_{k}\right| \leq b_{n}\right\}\right|=n\left|-E X_{k} I\left\{\left|X_{k}\right|>b_{n}\right\}\right| \leq n E|X| I\left\{|X|>b_{n}\right\} \\
& \leq \frac{n E X^{2} I\left\{|X|>b_{n}\right\}}{b_{n}}=\frac{(\log n)^{\alpha}}{\varepsilon} E X^{2} I\left\{|X|>b_{n}\right\}=o\left((\log n)^{\alpha}\right) \quad \text { as } \quad n \rightarrow \infty,
\end{aligned}
$$

so that, by applying the exponential bound as given in Gut (2007), Theorem 3.1.2, we obtain, for $n \geq n_{0}$ large,

$$
\begin{aligned}
P\left(A_{n, 1}^{\prime}\right) & =P\left(\left|S_{n}^{\prime}\right|>n \varepsilon\right) \leq P\left(\left|S_{n}^{\prime}-E S_{n}^{\prime}\right|>\left(n-\delta(\log n)^{\alpha}\right) \varepsilon\right) \\
& \left.\leq \exp \left\{-\frac{(\log n)^{\alpha}}{n \varepsilon} \cdot\left(n-\delta(\log n)^{\alpha}\right)\right) \varepsilon+\frac{(\log n)^{2 \alpha}}{n^{2} \varepsilon^{2}} \cdot n \operatorname{Var} X\right\} \\
& \leq c \exp \left\{-(\log n)^{\alpha}\right\},
\end{aligned}
$$

and, hence, that

$$
\sum_{n \geq n_{0}} \exp (\log n)^{\alpha} \frac{(\log n)^{\alpha-1}}{n^{2}} P\left(A_{n, 1}^{\prime}\right) \leq c \sum_{n \geq n_{0}} \frac{(\log n)^{\alpha-1}}{n^{2}}<\infty .
$$

- $P\left(A_{n, 2}^{\prime}\right)$

First note that

$$
n P\left(|X|>b_{n}\right) \leq n \cdot \frac{E \exp \left\{\left(\log ^{+}|X|\right)^{\alpha}\right\}}{\exp \left\{\left(\log b_{n}\right)^{\alpha}\right\}}
$$

which, together with the fact that for large $n$, say $n \geq n_{1}$,

$$
\left(\log b_{n}\right)^{\alpha}=(\log (\varepsilon+\log n-\alpha \log \log n))^{\alpha} \geq(1-\delta / 2)(\log n)^{\alpha},
$$

shows that

$$
n P\left(|X|>b_{n}\right) \leq c n \exp \left\{-(1-\delta / 2)(\log n)^{\alpha}\right\} \rightarrow 0 \quad \text { as } \quad n \rightarrow \infty .
$$

Next by (2.8) and (2.10) we have, for $n \geq n_{1}$,

$$
\begin{aligned}
P\left(A_{n, 2}^{\prime}\right) & \leq P\left(\left|S_{n-1}^{\prime}\right|>\varepsilon n-c_{n}\right) \cdot n P\left(|X|>b_{n}\right) \\
& =P\left(\left|S_{n-1}^{\prime}\right|>\varepsilon \delta n\right) \cdot n P\left(|X|>b_{n}\right) \leq c n \exp \left\{-\delta(\log n)^{\alpha}-(1-\delta / 2)(\log n)^{\alpha}\right\},
\end{aligned}
$$

which implies that

$$
\sum_{n \geq n_{1}} \exp \left\{(\log n)^{\alpha}\right\} \frac{(\log n)^{\alpha-1}}{n^{2}} P\left(A_{n, 2}^{\prime}\right) \leq c \sum_{n \geq n_{1}} \frac{(\log n)^{\alpha-1}}{n^{(1+\delta / 2)}}<\infty .
$$

- $P\left(A_{n}^{\prime \prime}\right)$

By (2.10) again,

$$
\sum_{n \geq n_{1}} \exp \left\{(\log n)^{\alpha}\right\} \frac{(\log n)^{\alpha-1}}{n^{2}} P\left(A_{n}^{\prime \prime}\right) \leq c \sum_{n \geq n_{1}} \exp \left\{-(1-\delta)(\log n)^{\alpha}\right\}(\log n)^{\alpha-1}<\infty .
$$

- $P\left(A_{n}^{\prime \prime \prime}\right)$

Since

$$
P\left(A_{n}^{\prime \prime \prime}\right) \leq n P\left(|X|>c_{n}\right)=n P(|X|>n \varepsilon(1-\delta)),
$$


it follows that

$$
\sum_{n=1}^{\infty} \exp \left\{(\log n)^{\alpha}\right\} \frac{(\log n)^{\alpha-1}}{n^{2}} P\left(A_{n}^{\prime \prime \prime}\right) \leq \sum_{n=1}^{\infty} \exp \left\{(\log n)^{\alpha}\right\} \frac{(\log n)^{\alpha-1}}{n} P(|X|>n \varepsilon(1-\delta)),
$$

and the latter sum converges iff $\varepsilon(1-\delta) \geq 1$ by Lemma 2.1 below.

By combining (2.6) with (2.9) - (2.12), we finally conclude that

$$
\sum_{n=1}^{\infty} \exp \left\{(\log n)^{\alpha}\right\} \frac{(\log n)^{\alpha-1}}{n^{2}} P\left(\left|S_{n}\right|>n \varepsilon\right)<\infty
$$

whenever $\varepsilon(1-\delta) \geq 1$, which, in view of the arbitrariness of $\delta$, finishes the proof of this step.

As for the remaining part of the proof, implications $(2.3) \Longrightarrow(2.2)$ and $(2.4) \Longrightarrow(2.2)$ are trivial, and $(2.2) \Longrightarrow(2.3)$ follows via an application of the Lévy inequalities. Finally $(2.2) \Longrightarrow$ (2.4) follows by mimicing the analogous part in the proof of Theorem 1.1 (cf. also Gut (2007), Section 7.12).

In order to prove the converse, more precisely that $(2.2) \Longrightarrow(2.1)$, one proceeds (with obvious modifications) along the lines of the analog for the classical Hsu-Robbins-Erdős theorem as provided in Gut (2007), page 314. The heart of the matter is to show that $P\left(\left|S_{n}\right|>n \varepsilon\right) \geq \frac{1}{2} n P(|X|>2 n \varepsilon)$, after which one applies the following lemma, in order to conclude that $E\left(\exp \left\{(\log (|X| /(2 \varepsilon)))^{\alpha}\right\}<\right.$ $\infty$, which, in turn, implies that $E\left(\exp \left\{(1-\delta)(\log |X|)^{\alpha}\right\}<\infty\right.$ for any $\delta>0$.

Lemma 2.1 For any random variable $X$ and $\gamma>0$,

$$
E \exp \left\{\left(\log ^{+}|X / \gamma|\right)^{\alpha}\right\}<\infty \Longleftrightarrow \sum_{n=1}^{\infty} \exp \left\{(\log n)^{\alpha}\right\} \frac{(\log n)^{\alpha-1}}{n} P(|X|>n \gamma)<\infty .
$$

Proof. The proof of the lemma is based on partial summation; cf. Gut (2007), Section 2.12 for results of this kind. We omit the details.

\section{Random fields}

Many of the earlier results in the area have been extended to multiindex models or random fields. The Kolmogorov strong law was extended to this setting by Smythe (1973). For the MarcinkiewiczZygmund analog we refer to Gut (1978). As an introductory example we quote the multiindex analog of the Baum-Katz Theorem 1.1 from Gut (1978), cf. Theorem 4.1 there.

In order to set the scene, let $\boldsymbol{Z}_{+}^{d}, d \geq 2$, denote the positive integer $d$-dimensional lattice with coordinate-wise partial ordering $\leq$, that is, for $\mathbf{m}=\left(m_{1}, m_{2}, \ldots, m_{d}\right)$ and $\mathbf{n}=\left(n_{1}, n_{2}, \ldots, n_{d}\right)$, $\mathbf{m} \leq \mathbf{n}$ means that $m_{k} \leq n_{k}$, for $k=1,2, \ldots, d$. The "size" of a point equals $|\mathbf{n}|=\prod_{k=1}^{d} n_{k}$, and $\mathbf{n} \rightarrow \infty$ means that $n_{k} \rightarrow \infty$, for all $k=1,2, \ldots, d$.

Theorem 3.1 Let $r>0, \alpha>1 / 2, \alpha r \geq 1$, and suppose that $\left\{X_{\mathbf{k}}, \mathbf{k} \in \boldsymbol{Z}_{+}^{d}\right\}$ are i.i.d. random variables with partial sums $S_{\mathbf{n}}=\sum_{\mathbf{k} \leq \mathbf{n}} X_{\mathbf{k}}, \mathbf{n} \in Z_{+}^{d}$. If

$$
E|X|^{r}\left(\log ^{+}|X|\right)^{d-1}<\infty \quad \text { and, if } r \geq 1, \quad E(X)=0,
$$

then

$$
\begin{aligned}
& \sum_{\mathbf{n}}|\mathbf{n}|^{\alpha r-2} P\left(\left|S_{\mathbf{n}}\right|>|\mathbf{n}|^{\alpha} \varepsilon\right)<\infty \quad \text { for all } \quad \varepsilon>0 \\
& \sum_{\mathbf{n}}|\mathbf{n}|^{\alpha r-2} P\left(\max _{\mathbf{k} \leq \mathbf{n}}\left|S_{\mathbf{k}}\right|>|\mathbf{n}|^{\alpha} \varepsilon\right)<\infty \quad \text { for all } \quad \varepsilon>0 .
\end{aligned}
$$

If $\alpha r>1$ we also have

$$
\sum_{j=1}^{\infty} j^{\alpha r-2} P\left(\left.\sup _{j \leq|\mathbf{k}|}\left|S_{\mathbf{k}} /\right| \mathbf{k}\right|^{\alpha} \mid>\varepsilon\right)<\infty \quad \text { for all } \varepsilon>0 .
$$

Conversely, if one of the sums is finite for all $\varepsilon>0$, then so are the others (for appropriate values of $r$ and $\alpha), E|X|^{r}\left(\log ^{+}|X|\right)^{d-1}<\infty$ and, if $r \geq 1, E(X)=0$. 
The corresponding results related to Theorems 2.1 and 1.2 , respectively, run as follows.

Theorem 3.2 Let $\alpha>1$, and suppose that $\left\{X_{\mathbf{k}}, \mathbf{k} \in \boldsymbol{Z}_{+}^{d}\right\}$ are i.i.d. random variables with $E(X)=$ 0 and partial sums $S_{\mathbf{n}}=\sum_{\mathbf{k} \leq \mathbf{n}} X_{\mathbf{k}}, \mathbf{n} \in \boldsymbol{Z}_{+}^{d}$. The following are equivalent:

$$
\begin{aligned}
& E \exp \left\{(\log |X|)^{\alpha}\right\}\left(\log ^{+}|X|\right)^{d-1}<\infty \\
& \sum_{\mathbf{n}} \exp \left\{(\log |\mathbf{n}|)^{\alpha}\right\} \cdot \frac{(\log |\mathbf{n}|)^{\alpha-1}}{|\mathbf{n}|^{2}} P\left(\left|S_{\mathbf{n}}\right|>|\mathbf{n}| \varepsilon\right)<\infty \quad \text { for all } \varepsilon>1 ; \\
& \sum_{\mathbf{n}} \exp \left\{(\log |\mathbf{n}|)^{\alpha}\right\} \cdot \frac{(\log |\mathbf{n}|)^{\alpha-1}}{|\mathbf{n}|^{2}} P\left(\max _{\mathbf{k} \leq \mathbf{n}}\left|S_{\mathbf{k}}\right|>|\mathbf{n}| \varepsilon\right)<\infty \quad \text { for all } \varepsilon>1 ; \\
& \sum_{j=1}^{\infty} \exp \left\{(\log j)^{\alpha}\right\} \cdot \frac{(\log j)^{\alpha-1}}{j^{2}} P\left(\sup _{j \leq|\mathbf{k}|}\left|S_{\mathbf{k}} /\right| \mathbf{k}||>\varepsilon\right)<\infty \quad \text { for all } \varepsilon>1 .
\end{aligned}
$$

Theorem 3.3 Let $0<\alpha<1$, and suppose that $\left\{X_{\mathbf{k}}, \mathbf{k} \in \boldsymbol{Z}_{+}^{d}\right\}$ are i.i.d. random variables with $E(X)=0$ and partial sums $S_{\mathbf{n}}=\sum_{\mathbf{k} \leq \mathbf{n}} X_{\mathbf{k}}, \mathbf{n} \in \boldsymbol{Z}_{+}^{d}$. The following are equivalent:

$$
\begin{aligned}
& E \exp \left\{|X|^{\alpha}\right\}\left(\log ^{+}|X|\right)^{d-1}<\infty ; \\
& \sum_{\mathbf{n}} \exp \left\{|\mathbf{n}|^{\alpha}\right\} \cdot|\mathbf{n}|^{\alpha-2} P\left(\left|S_{\mathbf{n}}\right|>|\mathbf{n}| \varepsilon\right)<\infty \quad \text { for all } \varepsilon>1 ; \\
& \sum_{\mathbf{n}} \exp \left\{|\mathbf{n}|^{\alpha}\right\} \cdot|\mathbf{n}|^{\alpha-2} P\left(\max _{\mathbf{k} \leq \mathbf{n}}\left|S_{\mathbf{k}}\right|>|\mathbf{n}| \varepsilon\right)<\infty \quad \text { for all } \varepsilon>1 ; \\
& \sum_{j=1}^{\infty} \exp \left\{j^{\alpha}\right\} \cdot j^{\alpha-2} P\left(\sup _{j \leq|\mathbf{k}|}\left|S_{\mathbf{k}} /\right| \mathbf{k}||>\varepsilon\right)<\infty \quad \text { for all } \quad \varepsilon>1 .
\end{aligned}
$$

REMARK 3.1 The equivalence with respect to the moment assumptions should be interpreted as in our earlier results.

The proofs of the theorems amount to rather straightforward generalizations of those in Gut $(1978,1980)$ and are omitted, except for the following extension of Lemma 2.1.

Lemma 3.1 For any random variable $X$ and $\gamma>0$,

$$
\begin{aligned}
& E \exp \left\{\left(\log ^{+}|X / \gamma|\right)^{\alpha}\right\}\left(\log ^{+}|X|\right)^{d-1}<\infty \\
& \Longleftrightarrow \sum_{\mathbf{n}} \exp \left\{(\log |\mathbf{n}|)^{\alpha}\right\} \frac{(\log |\mathbf{n}|)^{\alpha-1}}{|\mathbf{n}|} P(|X|>|\mathbf{n}| \gamma)<\infty \\
& E \exp \left\{|X / \gamma|^{\alpha}\right\}\left(\log ^{+}|X|\right)^{d-1}<\infty \Longleftrightarrow \sum_{\mathbf{n}} \exp \left\{|\mathbf{n}|^{\alpha}\right\} \cdot|\mathbf{n}|^{\alpha-1} P(|X|>|\mathbf{n}| \gamma)<\infty
\end{aligned}
$$

The basis of the proof of the lemma is, again, partial summation, together with the fact that terms with equisized indices are equal, viz., we may write

$$
\sum_{\mathbf{n}} \cdots=\sum_{j=1}^{\infty} \sum_{|\mathbf{n}|=j} d(j) \cdots
$$

where

$$
d(j)=\operatorname{Card}\{\mathbf{k}:|\mathbf{k}|=j\}, \quad j \geq 1 .
$$

Using this device the sums in the lemma turn into

$$
\begin{aligned}
\sum_{\mathbf{n}} \exp \left\{|\mathbf{n}|^{\alpha}\right\} \cdot|\mathbf{n}|^{\alpha-1} P(|X|>|\mathbf{n}|) & =\sum_{j=1}^{\infty} d(j) \exp \left\{j^{\alpha}\right\} \cdot j^{\alpha-1} P(|X|>j), \\
\sum_{\mathbf{n}} \exp \left\{(\log |\mathbf{n}|)^{\alpha}\right\} \frac{(\log |\mathbf{n}|)^{\alpha-1}}{|\mathbf{n}|} P(|X|>|\mathbf{n}|) & =\sum_{j=1}^{\infty} d(j) \exp \left\{(\log j)^{\alpha}\right\} \frac{(\log j)^{\alpha-1}}{j} P(|X|>j),
\end{aligned}
$$


respectivly, after it remains to connect these sums of the respective tail probabilities to the appropriate moment (cf. Gut (2007), Section 2.12).

In order to do so we also need the quantity

$$
M(j)=\operatorname{Card}\{\mathbf{k}:|\mathbf{k}| \leq j\} \quad\left(=\sum_{k=1}^{j} d(k)\right), \quad j \geq 1,
$$

with its asymptotics

$$
\frac{M(j)}{j(\log j)^{d-1}} \rightarrow \frac{1}{(d-1) !} \quad \text { as } \quad j \rightarrow \infty .
$$

For details concerning these number theoretical matters we refer to Hardy and Wright (1954), Chapter XVIII and to Titchmarsh (1951), relation (12.1.1) (for the case $d=2$ ).

\section{Further results and remarks}

So called "last exit times" related to the LLN and LIL have been investigated in various papers. The last exit time with respect to Theorem 1.2 would be

$$
L(\varepsilon)=\sup \left\{n:\left|S_{n}\right|>n \varepsilon\right\}
$$

for which we have the relation

$$
\{L(\varepsilon) \geq j\}=\left\{\sup _{k \geq j}\left|S_{k} / k\right|>\varepsilon\right\},
$$

which, in view of Theorem 1.2, tells us that, for $\varepsilon>0$,

$$
E \exp \left\{|X / \varepsilon|^{\alpha}\right\} \asymp \sum_{j=1}^{\infty} \exp \left\{j^{\alpha}\right\} \cdot j^{\alpha-2} P(L(\varepsilon) \geq j) .
$$

Using Theorem 1.2, together with a variation of Lemma 2.1, yields the following result.

Theorem 4.1 If $E(X)=0$ and $E \exp \left\{|X|^{\alpha}\right\}<\infty$ for some $\alpha \in(0,1)$, then

$$
E\left(\frac{\exp \left\{(L(\varepsilon))^{\alpha}\right\}}{L(\varepsilon)}\right)<\infty \text { for all } \varepsilon>1 \text {. }
$$

Conversely, if $E \frac{\exp \left\{(L(\varepsilon))^{\alpha}\right\}}{L(\varepsilon)}<\infty$ for some $\varepsilon>0$, then $E \exp \left\{\left|X / \varepsilon^{\prime}\right|^{\alpha}\right\}<\infty$ for any $\varepsilon^{\prime}>\varepsilon$.

Turning our attention to Theorem 2.1, we obtain, in essence,

$$
E \exp \left\{(\log |X|)^{\alpha}\right\} \asymp \sum_{j=1}^{\infty} \exp \left\{(\log j)^{\alpha}\right\} \frac{\left((\log j)^{\alpha-1}\right.}{j^{2}} P(L(\varepsilon) \geq j),
$$

and combining this with Lemma 2.1 we arrive at

Theorem 4.2 If $E(X)=0$ and $E \exp \left\{(\log |X|)^{\alpha}\right\}<\infty$ for some $\alpha>1$, then

$$
E\left(\frac{\exp \left\{\left(\log (L(\varepsilon))^{\alpha}\right\}\right.}{L(\varepsilon)}\right)<\infty \text { for all } \varepsilon>1
$$

Conversely, if $E\left(\frac{\exp \left\{\left(\log (L(\varepsilon))^{\alpha}\right\}\right.}{L(\varepsilon)}\right)<\infty$ for some $\varepsilon>0$, then $E \exp \left\{(1-\delta)(\log |X|)^{\alpha}\right\}<\infty$ for any $\delta>0$.

We conclude by mentioning without any details that corresponding results may be stated for

$\diamond$ random fields; one considers $L_{d}(\varepsilon)=\sup \left\{|\mathbf{n}|:\left|S_{\mathbf{n}}\right|>|\mathbf{n}| \varepsilon\right\} ;$

$\diamond$ the counting variable $N_{d}(\varepsilon)=\mathcal{C}$ ard $\left\{|\mathbf{n}|:\left|S_{\mathbf{n}}\right|>|\mathbf{n}| \varepsilon\right\}$.

For the case of polynomial growth we refer to Gut (1980), Section 8, and further references given there. 


\section{Acknowledgement}

The results of this paper were essentially worked out during the first authors visit at the Department of Number Theory and Probability Theory at the University of Ulm. The same author (A.G.) wishes to thank his coauthor (U.S.) for providing a most stimulating and inspiring stay in Ulm, as well for his kind and generous hospitality. He also wishes to thank the University of Ulm for financial support.

\section{References}

[1] Baum, L.E., Katz, M., 1965. Convergence rates in the law of large numbers. Trans. Amer. Math. Soc. 120, 108-123.

[2] Chen, R. (1978). A remark on the tail probability of a distribution. J. Multivariate Analysis 8, 328-333.

[3] Chow, Y.S. (1973). Delayed sums and Borel summability of independent, identically distributed random variables. Bull. Inst. Math. Acad. Sinica 1, 207-220.

[4] Erdős, P. (1949). On a theorem of Hsu and Robbins. Ann. Math. Statist. 20, 286-291.

[5] Erdős, P. (1950). Remark on my paper "On a theorem of Hsu and Robbins". Ann. Math. Statist. 21, 138.

[6] Gut, A., 1978. Marcinkiewicz laws and convergence rates in the law of large numbers for random variables with multidimensional indices. Ann. Probab. 6, 469-482.

[7] Gut, A., 1980. Convergence rates for probabilities of moderate deviations for sums of random variables with multidimensional indices. Ann. Probab. 8, 298-313.

[8] Gut, A., 2007. Probability: A Graduate Course, Corr. 2nd printing. Springer-Verlag, New York.

[9] Gut, A., and Spătaru, A. (2000). Precise asymptotics in the Baum-Katz and Davis law of large numbers. J. Math. Anal. Appl. 248, 233-246.

[10] Hardy, G.H., Wright, E.M., 1954. An Introduction to the Theory of Numbers, 3rd ed. Oxford University Press.

[11] Heyde, C.C. (1975). A supplement to the strong law of large numbers. J. Appl. Probab. 12, 173-175.

[12] Hsu, P.L., Robbins, H., 1947. Complete convergence and the law of large numbers. Proc. Nat. Acad. Sci. USA 33, 25-31.

[13] Lai, T.L., 1974. Limit theorems for delayed sums. Ann. Probab. 2, 432-440.

[14] Lanzinger, H., 1998. A Baum-Katz theorem for random variables under exponential moment conditions. Statist. Probab. Lett. 39, 89-95.

[15] Katz, M. (1963). The probability in the tail of a distribution. Ann. Math. Statist. 34, 312-318.

[16] Smythe, R., 1973. Strong laws of large numbers for $r$-dimensional arrays of random variables. Ann. Probab. 1, 164-170.

[17] Spătaru, A. (1999). Precise asymptotics in Spitzer's law of large numbers. J. Theoret. Probab. $12,811-819$.

[18] Spitzer, F. (1956). A combinatorial lemma and its applications to probability theory. Trans. Amer. Math. Soc. 82, 323-339.

[19] Titchmarsh, E.C., 1951. The Theory of the Riemann Zeta-function, 2nd ed. Oxford University Press. 
Allan Gut, Department of Mathematics, Uppsala University, Box 480, SE-751 06 Uppsala, Sweden; Email: allan.gut@math.uu.se

URL: http://www.math.uu.se/ ${ }^{\text {allan }}$

Ulrich Stadtmüller, Ulm University, Department of Number Theory and Probability Theory, D-89069 Ulm, Germany;

Email: ulrich.stadtmueller@uni-ulm.de

URL: http://www.mathematik.uni-ulm.de/matheIII/members/stadtmueller/stadtmueller.html 